Research Paper:

\title{
Caregivers' Perspective About the Levels of Sensory Processing Problems in Children With Autism
}

\author{
Mohannad Mohammad Husni Amireh ${ }^{1 *}$ (D), Fatma El-Zahraa Ahmed Mahmoud Omer ${ }^{2}$ (i)
}

1. Department of Occupational Therapy, Launch Egypt Center for Special Needs, Cairo, Egypt.

2. Launch Egypt Center for Special Needs, Cairo, Egypt.

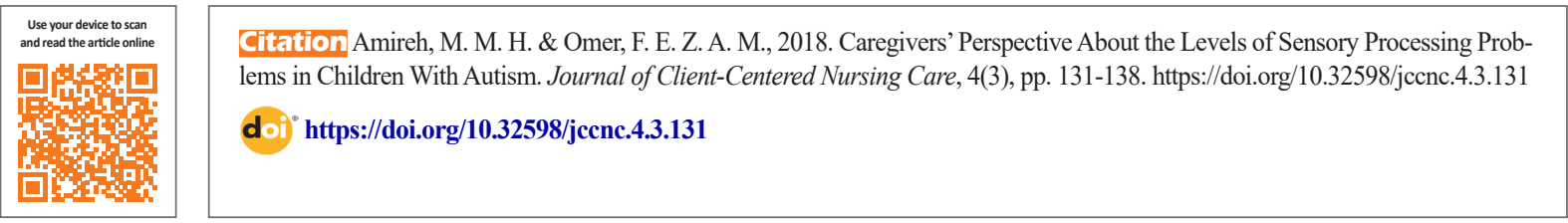

\section{(1) (8)}

Article info:

Received: 10 Feb 2018

First Revision: 28 Feb 2018

Accepted: 23 Jun 2018

Published: 01 Aug 2018
Keywords:

Autism, Sensory

processing, Adaptive

behavior, Occupational

therapy

\begin{abstract}
A B S T RA C T
Background: Children with autism should interact and get along with their surrounding environment. Therefore, the researchers must try to explain the reasons of challenges in their adaptive behaviors. This study aimed to evaluate levels of challenges in sensory processing and the motor domain of adaptive behavior among children with autism in Egypt, and to explore the effect of sensory processing on subdomains of gross and fine motor skills.

Methods: This is a cross-sectional study. Study questionnaires were distributed to 180 caregivers of children with autism in Cairo, Egypt, between July 2017 and February 2018. The caregivers were selected by convenience sampling method. The questionnaires used were the sensory checklist and adaptive behavior scales. Descriptive statistics and comparing means were used to determine the levels of sensory processing and motor domain of adaptive behavior. Linear regression analyses was used to test the effects of sensory domains on motor areas. SPSS-PC V. 25 was used to analyze the obtained data.
\end{abstract}

Results: The results demonstrated various problems in children's sensory processing and the motor domain of adaptive behavior. Caregivers reported that $96.67 \%$ of the children had frequent sensory challenges in at least one component of sensory domains, mostly in visual system (27.2\%). Subdomain of fine motor skills (Mean=1.4951) showed higher challenges than gross motor skills (Mean=1.9455). This study proved significant inverse effect for movement processing on gross motor subdomain at $\alpha=0.05$.

Conclusion: Parents of children with autism, occupational therapists, and other healthcare practitioners should gain in-depth knowledge regarding their children's behaviors and their possible reasons.

\section{* Corresponding Author:}

Mohannad Mohammad Husni Amireh, PhD.

Address: Department of Occupational Therapy, Launch Egypt Center for Special Needs, Cairo, Egypt.

Tel: +20 (112) 6487347

E-mail: mohannad.amireh@yahoo.com 


\section{Highlights}

- About $96.67 \%$ of children with autism have sensory challenges in at least one sensory domain.

- Almost $74.4 \%$ of these children got scores more than 2 SD below the typical mean of Vineland scores.

- The subdomain of fine motor skills in these children showed higher challenges than gross motor skills.

- This study proved significant inverse effect for the movement processing on gross motor skills of autism children.

\section{Plain Language Summary}

Autism is a disorder that limits persons' ability to interact with others. It affects social communication, language, and sensory processing. Autism is characterized by many manifestations, one of them is sensory processing disorder, which refers to unusual receiving, organizing, and interpreting sensory stimuli. Sensory processing disorder may be over-responsivity, under-responsivity, or sensory seeking. Sensory processing disorder affects adaptive behavior i.e. a person's ability to function independently. This study aimed to evaluate challenges in sensory processing and the motor domain of adaptive behavior among children with autism in Egypt, and also to explore the effect of sensory processing on subdomains of gross and fine motor skills. The results showed various challenges in children's sensory processing and the motor domain of adaptive behavior.

\section{Background}

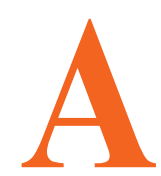

merican Psychiatric Association has defined autism as an early-onset neurological disease characterized by difficulties with social communication and social interaction. In addition, autistic person has two or more of the symptoms of limited interests and repetitive behaviors, including stereotyped/repetitive speech, motor movements, use of objects, over-adherence to routines, ritualized verbal or nonverbal behaviors, or over-resistance to change. Moreover, there are highly limited, fixated interests abnormal with regard to the intensity of focus whether hyper- or hypo-reactivity to sensory input or unusual interest in sensory aspect of the environment (American Psychiatric Association 2011).

A lot of emphasis has been placed on studying functional outcomes in persons with autism. The term "adaptive behavior" is used to indicate a person's ability to function independently in his or her environment. Most of the tools assessing adaptive behavior contain different subscales, for example; the Vineland Adaptive Behavior Scales-3 ${ }^{\text {th }}$ Edition (Vineland-III) contains five domains; "communication domain", "daily living skills domain", "socialization domain", "motor skills domain", and "maladaptive behavior domain" (Sparrow, Cicchetti \& Saulnier 2016).
Communication domain includes receptive, expressive, and written subdomains. Communication subdomains assess person's listening, paying attention, understanding, talking, using words and sentences to gather and provide information, understanding how letters make words, and understanding reading and writing. Daily living skills domain comprised personal, domestic, and community subdomains that assess person's ability to eat, dress, practice personal hygiene, perform household tasks, and use time, money, phone, computer, and job skills. Socialization domain assesses person's interaction with others, playing and leisure, and demonstration responsibility and sensitivity to others.

Socialization domain contained subdomains of interpersonal relationships, play and leisure, and coping skills. Domain of motor skills includes gross motor and fine motor subdomains. Gross motor subdomain assesses the person's ability to use arms and legs for movement and coordination. Fine motor subdomain assesses the person's ability to use hands and fingers to manipulate objects. Subdomains of internalizing, externalizing, and critical items constitute the domain of maladaptive behavior (Sparrow, Cicchetti \& Saulnier 2016).

Participating in everyday activities can be deteriorated by many causes, one of which is sensory processing disorder. Miller and Lane (2000) defined sensory processing as receiving, organizing, and interpreting of sensory input by the seven sensory systems; tactile, vestibular, 
proprioceptive, visual, auditory, gustatory, and olfactory. Sensory processing disorder is referred to unusual responses to sensory stimuli, such as over-responsivity or under-responsivity (Gabriels et al. 2005; Dawson \& Watling 2000). Sensory processing disorder may also be sensory seeking (Lane et al. 2010).

Byrne (2009) mentioned that nurse practitioners unfortunately had limited access to information about sensory processing disorders; nursing and medical curricula have rarely paid attention to sensory processing disorders and research about the roles of nurse practitioners about persons diagnosed with sensory processing disorders are limited. Nurse practitioners as the members of health care team should know about sensory processing disorders because they see the children first, have direct communication with their parents, and refer them to other healthcare practitioners.

This study aimed to detect sensory processing challenges and adaptation skills in the motor domain of adaptive behavior in children with autism in Egypt. Also, it explored the effect of sensory processing problems on subdomains of fine motor and gross motor skills, as there were limited studies assessing this relationship.

Some studies have evaluated sensory processing and adaptive behavior, and explores their relationship in autistic people. O'Donnell et al. (2012) studied sensory processing disorders and their relationships with problem behavior, adaptive behavior, and cognitive function in preschool children with autism in Washington. The researchers concluded that most of autistic children had sensory processing challenges; $60.7 \%$ of autistic children were in category of definite difference, and $17.9 \%$ in probable difference category. The area of tactile had the highest mean (26.9) of problems, and the area taste/ smell sensitivity had the lowest one (12.3).

Most of autistic children got low Vineland scores; $74.4 \%$ had scores more than $2 \mathrm{SD}$ below the typical mean, and $25.6 \%$ of the sample had scores more than $1 \mathrm{SD}$ below the typical mean (typical mean=100, and $\mathrm{SD}=15)$. There were no significant relationships between sensory processing challenges and total scores and adaptive behavior although there were weak association between higher scores of sensory processing and lower scores of adaptive behavior. Nieto, Lopez and Gandia (2017) studied sensory processing patterns in autistic children in Spain. They examined the relationship between atypical sensory processing and adaptive behavior domains (except motor area), and between sensory subtypes and maternal stress. To achieve the objectives of the study, the researchers used Short Sensory Profile-II (SSP-II) to assess sensory processing, Vineland Adaptive Behavior Scales (VABS) to assess adaptive behaviors, and Parenting Stress Index Short Form (PSI-SF) to assess parenting stress.

The results showed that $86.7 \%$ of the sample had atypical sensory features distributed on the different quadrants; 28.98\% were "sensor", 25.22\% were "avoider", $16.91 \%$ were "seeker", and $15.69 \%$ were "bystander". Moreover, there were no significant correlations between atypical sensory processing and adaptive behavior except the category of maladaptive behavior which showed significant positive correlation with all scores of the SSP-II.

Baker et al. (2008) investigated sensory processing challenges and their relationships with domains of adaptive behavior except motor, and with developmental behavior, in children with autism in Australia. SSP, VABS, and Developmental Behavior Checklist-Parent (DBC-P) were used to achieve the study objectives. DBC-P was used to assess behavioral and emotional problems. Based on the results, $82 \%$ of the samples had either a probable or definite difference in sensory processing. Under-responsive/seeks sensation and auditory filtering areas had the highest disorder $(68 \%$ of the sample for each).

The results indicated strong negative correlations between SSP scores and the domain of maladaptive behavior, which means that the poorer sensory processing ability correlates with the higher levels of behavioral and or emotional problems. Therefore, there was a moderate positive correlation between total score of SSP and the mean score of the daily living skills, which deteriorate with low ability of sensory processing.

Lane et al. (2010) examined sensory processing and adaptive behavior in children with autism in Australia. SSP and VABS were used to assess the study objectives. Overall, $87 \%$ of the sample demonstrated sensory processing challenge. Autistic children demonstrated the highest challenge in the area of tactile sensitivity, and the lowest in the area of taste/smell sensitivity. The results also proved significant inverse association between tactile, taste/smell, under-responsive/seeks sensation, auditory filtering and visual/auditory sensitivity and maladaptive behavior at $\alpha=0.001$. In other words, the lower sensory processing is associated with the higher challenge in the presentation of maladaptive behavior. 
Jasmin et al. (2008) studied correlations between sensory processing, motor skills, daily living skills, and adaptive behavior, in preschool children with autism spectrum disorder in Montreal. The researchers used Peabody Developmental Motor Scales-II (PDMS-II) to assess motor skills, Functional Independence Measure for children (WeeFIM) to assess daily living skills, as well as SP and VABS-II. About $94 \%$ of the samples showed atypical responses in at least one item of the sensory processing. Also, $54.5 \%$ of children showed "sensory seeking", 54.5\% "sensory sensitivity", 48.5\% "low registration", and 39.4\% "sensory avoiding". The results also indicate positive and significant correlation between sensory avoiding and subdomain of fine motor skills in VABS-II.

This study investigated sensory processing challenges in domains of movement, visual system, eye contact, touch, food, hearing, smell, and sleep in children with autism in Egypt. It also evaluated adaptive levels of fine and gross motor skills among these children. Finally, it studied the effect of sensory processing domains on fine and gross motor skills.

\section{Materials and Methods}

It was a cross-sectional survey to study adaptive levels of fine and gross motor skills in children with autism, and explore the effects of sensory processing disorders on these levels. The study population were caregivers of children who diagnosed with autism in Cairo, Egypt. A convenience sampling technique was used to select the samples. A total of 280 caregivers received questionnaires in centers of special needs in the governorate of Cairo in Egypt between July 2017 and February 2018. The returned questionnaires were 185 , and 5 questionnaires were excluded because they missed the child's diagnosis.

The questionnaires were based on the sensory checklist designed to identify and understand children's profile of sensory issues (Larkey 2007), and the adaptive behavior Scales to evaluate the children's adaptive behavior (Abd El-Samee 2016). The sensory checklist contained 117 items, divided according to eight subscales as the following: 22 items for the domain of movement, 15 items for visual system, 10 items for eye contact with people and objects, 32 items for touch, 14 items for food, 11 items for hearing, 8 items for smell, and 5 items for sleep (Larkey 2007). In terms of sensory processing issues, caregivers responded to each item using a 3-point Likert scale whereby $1=$ rarely, $2=$ sometimes, and $3=$ frequently.
The items of sensory checklist were translated into Arabic. In order to test the comprehensibility, logic, and ambiguity of the items, 25 questionnaires were distributed for a pilot study. Adaptive behavior scales has 4 domains, including motor, conceptual, practical, and social domain. In this study, just motor domain was used that included skills of gross motor and fine motor. The part of gross motor skills contained 24 items, and the part of fine motor skills contained 20 items (Abd El-Samee 2016). Items of adaptive behavior scales were validated in Arabic and English languages by the author (Abd ElSamee 2016).

In terms of gross and fine motor skills, caregivers answered each item on a 4-point Likert scale, whereby $0=$ unable to do the behavior, $1=$ able to do the behavior, but never or almost never do it when needed, or on his/her own without being reminded, $2=$ only does it sometimes when needed, and $3=$ able to perform the behavior, and do it most of the time without being reminded, or did it at younger age, but has now outgrown it. SPSS V. 25.0 was used to analyze the data. Descriptive statistics were used to present sensory processing and motor adaptive levels. Regression analyses was used to calculate the effect of each sensory area (as independent variables) on gross and fine motor skills (as dependent variables). The caregivers received the information sheet explaining the purpose, method, risks, and benefits of this study before their participation. They were allowed to leave the study whenever they want and their data would be kept confidential.

\section{Results}

Table 1 presents the descriptive statistics of the samples regarding their age, education degree, and their relationship with the child, and child's gender. About 53.9\% of the participants were 25 to 35 years old and $31.7 \%$ were 35 to 45 years old. About, $43.3 \%$ were mothers, and $37.8 \%$ were specialists working with the child. Most participants $(73.3 \%)$ had bachelor degree. Of the children with autism, $75.6 \%$ were males and $24.4 \%$ females. Regarding the children's ages, $20.6 \%$ were $\leq 4$ years old, $36.6 \%$ were $4-8$ years old, $25.0 \%$ were $8-12$ years old, and $17.8 \%$ were more than 12 years old.

Table 2 presents the mean score of sensory processing in each domain. Comparing the means, visual system got the highest mean score of sensory challenges (1.76), and smell the lowest mean score (1.34). About $60 \%$ of the children demonstrated some problems in at least one domain of sensory processing. Number of cases that had means between 2 and 3 regarding sensory processing 
Table 1. Descriptive Statistics of study participants (Caregivers and children)

\begin{tabular}{|c|c|c|c|c|c|c|}
\hline Featu & & 18 - 24 Years & 25 - 35 Years & & $\begin{array}{c}35-45 \\
\text { Years }\end{array}$ & $>45$ Years \\
\hline Age of the care- & Frequency & 9 & 97 & & 57 & 17 \\
\hline & Percentage & 5 & 53.9 & & 31.7 & 9.4 \\
\hline Fea & ure & Illiterate & Primary School & High School & Bachelor & Master or PhD. \\
\hline Participant's degre & Frequency & 2 & 1 & 12 & 132 & 33 \\
\hline & Percentage & 1.1 & 0.6 & 6.7 & 73.3 & 18.3 \\
\hline & Featur & & & Male & & Female \\
\hline & & Frequ & & 136 & & 44 \\
\hline Gender of th & children & Perce & & 75.6 & & 24.4 \\
\hline Fea & & Father & Mother & her/Sister & Specialist & Other \\
\hline Relationship with & Frequency & 22 & 78 & 10 & 68 & 2 \\
\hline the child & Percentage & 12.2 & 43.3 & 5.6 & 37.8 & 1.1 \\
\hline
\end{tabular}

are presented in Table 3. The domain of visual system had the highest number with $49(27.2 \%)$ children. Just $15(8.3 \%)$ children had means greater than 2 in the domain of touch. Around $96.67 \%$ of the samples had a frequent sensory challenge in at least one item.

Descriptive statistics of motor domain of adaptive behavior were also calculated using means and standard deviations (Table 4). Higher mean is associated with less ability to do the behavior. Table 5 presents the percentages of the children who had mean scores $\leq 1$ in the motor domain; $4.4 \%$ in gross motor skills, and $27.2 \%$ in fine motor skills. Linear regression at $\alpha=0.05$ was used to test the effects of the sensory domains on the motor areas. Results are presented in Table 6. There was a significant inverse effect for movement on gross motor skills; the higher challenges in the domain of movement, the less ability to do the behaviors that involves using arms and legs for movement and coordination to adapt.

\section{Discussion}

This study concluded that $60 \%$ of children with autism experienced sensory challenges in at least one sensory domain. This result is consistent with O'Donnell et al. (2012) who concluded that $60.7 \%$ of autistic children were in the category of definite difference regarding sensory processing. This study also concluded that $96.67 \%$ of the children had a frequent behavior indicating sensory challenges in one or more items. This result resembled that of Jasmin et al. (2009) who concluded that $94 \%$ of

Table 2. Means and SD of sensory categories

\begin{tabular}{ccccccccc}
\hline Measures & MM & MVS & MEC & MT & MF & MH & MSm & 1.3403 \\
\hline Mean & 1.6588 & 1.7611 & 1.6883 & 1.4800 & 1.4393 & 1.6672 & 1.4733 \\
N & 180 & 180 & 180 & 180 & 180 & 180 & 0.48622 & 0.42076 \\
SD & 0.36640 & 0.39222 & 0.47804 & 0.32212 & 0.34039 & 0.49530 \\
\hline
\end{tabular}

Abbreviations: MM: Mean of items of "Movement"; MVS: Mean of items of "Visual System"; MEC: Mean of items of "Eye Contact"; MT: Mean of items of "Touch"; MF: Mean of items of "Food"; MH: Mean of items of "Hearing"; MSm: Mean of items of "Smell"; MSl: Mean of items of "Sleep" 
Table 3. Number of cases who had means greater or equal than 2 in sensory domains

\begin{tabular}{cll}
\hline & Sensory Dimension & \\
\hline Sensory Sub-Domains & No. & $\%$ \\
\hline MM & 33 & 18.3 \\
\hline MVS & 49 & 27.2 \\
\hline MEC & 46 & 25.6 \\
MT & 15 & 8.3 \\
MF & 18 & 10.0 \\
MH & 46 & 25.6 \\
MSm & 17 & 9.4 \\
MSI & 33 & 18.3 \\
\hline
\end{tabular}

Client- Centered Nursing Care

Abbreviations: MM: Mean of items of "Movement"; MVS: Mean of items of "Visual System"; MEC: Mean of items of "Eye Contact"; MT: Mean of items of "Touch"; MF: Mean of items of "Food"; MH: Mean of items of "Hearing"; MSm: Mean of items of "Smell"; MSl: Mean of items of "Sleep"

Table 4. Means and standard deviations of motor category of adaptive behavior

\begin{tabular}{ccc}
\hline Measures & MGM & MFM \\
\hline Mean & 1.9455 & 1.4951 \\
N & 180 & 180 \\
SD & 0.53081 & 0.64260 \\
\hline
\end{tabular}

Abbreviations: MGM: Mean of items of "Gross Motor", MFM: Mean of items of "Fine Motor" Client- Centered Nursing Care

autistic children had atypical responses in at least one domain of the sensory processing.

The results indicated that the highest mean of sensory challenge was in the domain of visual system, but contradicted the other studies concluding that it was in the domain of the tactile system (O'Donnell et al. 2012; Lane et al. 2010), or under-responsive/seeks sensation and auditory filtering (Baker et al. 2008). The area of smell had the lowest challenge among areas of sensory processing, and this result resembled those of O'Donnell et al. (2012), Lane et al. (2010), and Baker et al. (2008).
This study also concluded that the domain of movement had a significant inverse effect on the subdomain of gross motor skills. Previous studies had different results regarding the effect of sensory processing on adaptive behavior in children with autism. O'Donnell et al. (2012) did not report this effect. In addition, Nieto, Lopez and Gandia (2017) did not find an effect, with note that the researchers excluded the motor domain. Also, Baker et al. (2008) excluded the motor domain in their study, but their results indicated strong inverse effects of sensory challenges on the domain of maladaptive behavior, and moderate positive effect on the domain of daily living skills.

Table 5. Number of cases who had mean scores lower or equal to 1 in motor subdomains

\begin{tabular}{|c|c|c|}
\hline \multicolumn{3}{|c|}{ Motor Dimension } \\
\hline Sub-Domains of Motor Component of Adaptive Behavior & No. & $\%$ \\
\hline MGM & 8 & 4.4 \\
\hline MFM & 49 & 27.2 \\
\hline
\end{tabular}

Abbreviations: MGM: Mean of items of "Gross Motor", MFM: Mean of items of "Fine Motor"

Client- Centered Nursing Care 
Table 6. Regression analysis of sensory domains on motor skills

\begin{tabular}{|c|c|c|c|c|c|}
\hline \multirow{3}{*}{ Model } & \multicolumn{4}{|c|}{ Regression Analysis of Sensory Domains on Gross Motor Skills Coefficients ${ }^{a}$} & \multirow{3}{*}{ Sig. } \\
\hline & \multicolumn{2}{|c|}{ Unstandardized Coefficients } & \multicolumn{2}{|c|}{ Standardized Coefficients } & \\
\hline & B & Std. Error & Beta & $\mathbf{t}$ & \\
\hline (Constant) & 2.642 & 0.237 & - & 11.129 & 0.000 \\
\hline MM & -0.363 & 0.134 & -0.251 & -2.718 & 0.007 \\
\hline MVS & -0.105 & 0.125 & -0.078 & -0.837 & 0.404 \\
\hline MEC & -0.129 & 0.110 & -0.116 & -1.171 & 0.243 \\
\hline MT & 0.283 & 0.184 & 0.172 & 1.537 & 0.126 \\
\hline MF & -0.129 & 0.154 & -0.083 & -0.839 & 0.402 \\
\hline $\mathrm{MH}$ & -0.008 & 0.091 & -0.007 & -0.087 & 0.931 \\
\hline MSm & 0.018 & 0.101 & 0.014 & 0.177 & 0.860 \\
\hline MSI & 0.044 & 0.091 & 0.041 & 0.485 & 0.628 \\
\hline \multicolumn{6}{|c|}{ a. Dependent Variable: MGM } \\
\hline
\end{tabular}

\begin{tabular}{|c|c|c|c|c|c|}
\hline \multirow{3}{*}{ Model } & \multicolumn{4}{|c|}{ Regression Analysis of Sensory Domains on Fine Motor Skills Coefficients ${ }^{a}$} & \multirow{3}{*}{ Sig. } \\
\hline & \multicolumn{2}{|c|}{ Unstandardized Coefficients } & \multicolumn{2}{|c|}{ Standardized Coefficients } & \\
\hline & B & Std. Error & Beta & $\mathbf{t}$ & \\
\hline (Constant) & 2.293 & 0.284 & & 8.076 & 0.000 \\
\hline MM & -0.381 & 0.160 & -0.217 & -2.385 & 0.018 \\
\hline MVS & -0.218 & 0.150 & -0.133 & -1.454 & 0.148 \\
\hline MEC & -0.168 & 0.131 & -0.125 & -1.279 & 0.203 \\
\hline MT & 0.389 & 0.220 & 0.195 & 1.769 & 0.079 \\
\hline MF & -0.160 & 0.184 & -0.085 & -0.867 & 0.387 \\
\hline $\mathrm{MH}$ & 0.055 & 0.109 & 0.041 & 0.502 & 0.616 \\
\hline MSm & 0.115 & 0.120 & 0.075 & 0.958 & 0.340 \\
\hline \multirow[t]{2}{*}{ MSI } & -0.061 & 0.109 & -0.047 & -0.561 & 0.576 \\
\hline & \multicolumn{4}{|c|}{ a. Dependent Variable: MFM } & \\
\hline
\end{tabular}

Client- Centered Nursing Care

Abbreviations: MM: Mean of items of "Movement"; MVS: Mean of items of "Visual System"; MEC: Mean of items of "Eye Contact"; MT: Mean of items of "Touch"; MF: Mean of items of "Food"; MH: Mean of items of "Hearing"; MSm: Mean of items of "Smell"; MSl: Mean of items of "Sleep", MGM: Mean of items of "Gross Motor", MFM: Mean of items of "Fine Motor"

Lane et al. (2010) found strong inverse effects of challenges in all sensory areas on the maladaptive behavior. Jasmin et al. (2009) concluded positive significant correlation between sensory avoiding and subdomain of fine motor skills. This study demonstrated the levels of sensory challenges and adaptive behavior experienced by children with autism in Egypt, and revealed the effect of sensory processing on motor domain of adaptive behavior among them. Limited research has been conducted in Egypt on this issue but understanding the levels of sensory challenges and adaptive behaviors helps specialists 
and parents to deal with children better and understand these issues more deeply.

It is highly recommended to introduce sensory processing disorder for nurse practitioners through formal and informal education such as curricula, research studies, and interdisciplinary meetings with other healthcare practitioners. More studies should be conducted to investigate the levels of sensory challenges and their effect on all domains of adaptive behavior in children with special needs in Egypt and other countries.

\section{Ethical Considerations}

\section{Compliance with ethical guidelines}

The caregivers received an information sheet describing the purpose, method, the risks, and benefits of this study before participation. They were informed that they had the right of refusing participation, and the data they provided would remain private and confidential in case of participating.

\section{Funding}

This research did not receive any specific grant from funding agencies in the public, commercial, or not-forprofit sectors.

\section{Authors contributions}

Conceptualization: Mohannad Amireh; Collecting data: Fatma Omer; Investigation: Fatma Omer; WritingOriginal Draft: Mohannad Amireh; Writing-Review \& Editing: Mohannad Amireh; Funding Acquisition: All authors; and Supervision: Mohannad Amireh.

\section{Conflicts of interest}

The authors declared no conflict of interest.

\section{Acknowledgements}

The researchers are profoundly thankful to all managers and practitioners in the Centers of Special Needs who helped us complete this study.
American Psychiatric Association., 2011. Diagnostic and stical manual of mental disorders. Washington: American Psychiatric Association Publishing.

Baker, A. E., Lane, A., Angley, M. T. \& Young, R. L., 2008. The relationship between sensory processing patterns and behavioural responsiveness in autistic disorder: A pilot study. Journal of Autism and Developmental Disorders, 38(5), pp. 867-75. [DOI:10.1007/s10803-007-0459-0] [PMID]

Byrne, M. W., 2009. Sensory processing disorder: Any of a nurse practitioner's business. Journal of the American Academy of Nurse Practitioners, 21(6), pp. 314-21. [DOI:10.1111/j.17457599.2009.00417.x] [PMID]

Dawson, G. \& Watling, R., 2000. Interventions to facilitate auditory, visual, and motor integration in autism: A review of the evidence. Journal of Autism and Developmental Disorders, 30(5), pp. 415-21. [DOI:10.1023/A:1005547422749]

Gabriels, R.L., et al., 2005. Repetitive behaviors in autism: Relationships with associated clinical features. Research in Developmental Disabilities, 26(2), pp. 169-81. [DOI:10.1016/j. ridd.2004.05.003] [PMID]

Jasmin, E., et al., 2009. Sensori-motor and daily living skills of preschool children with autism spectrum disorders. Journal of Autism and Developmental Disorders, 39(2), pp. 231-41. [DOI:10.1007/s10803-008-0617-z] [PMID]

Lane, A. E., et al., 2010. Sensory processing subtypes in autism: Association with adaptive behavior. Journal of Autism and Developmental Disorders, 40(1), pp. 112-22. [DOI:10.1007/s10803009-0840-2] [PMID]

Larkey, S., 2007. Practical Sensory Programmes: For Students with Autism Spectrum Disorder and Other Special Needs. London: Jessica Kingsley Publishers.

Miller, L. \& Lane, S. J., 2000. Toward a consensus in terminology in sensory integration theory and practice: Part 1: Taxonomy of neurophysiological processes. Sensory Integration Special Interest Section Quarterly, 23(1), pp. 1-4.

Nieto, C., Lopez, B. \& Gandia, H., 2017. Relationships between atypical sensory processing patterns, maladaptive behaviour and maternal stress in Spanish children with autism spectrum disorder. Journal of Intellectual Disability Research, 61(12), pp. 1140-50. [DOI:10.1111/jir.12435] [PMID]

O'Donnell, S., et al., 2012. Sensory processing, problem behavior, adaptive behavior, and cognition in preschool children with autism spectrum disorders. American Journal of Occupational Therapy, 66(5), pp. 586-94. [DOI:10.5014/ajot.2012.004168] [PMID]

Sparrow, S. S., Cicchetti, D. V. \& Saulnier, C. A., 2016. Vineland Adaptive Behavior Scales, 3rd Edition (Vineland-3). London: Pearson.

\section{References}

Abd El Samee, A. E., 2016. Adaptive behavior scales. Cairo: Arab Corporation for Psychological Tests. 